Neonatal data included gestational age, birth weight, gender, mode of delivery, Apgar scores at 1 and 5 minutes of life, early or late onset sepsis, necrotizing enterocolitis (NEC), intraventricular haemorrhage (IVH), TORCH infections, asphyxia, bleeding site, were collected. The likely cause of NT, Day of life (DOL) of onset of NT, resolution $>150000$, Nadir of platelet count (mean) and severity of NT were collected. Early onset NT is onset $<72$ hours and late onset NT as onset $>72$ hours.

Results Total of 5196 live-born neonates were studied, 73 $(0.014 \%)$ were found to have NT. The incidence was higher among Very Low Birth Weight (VLBW, <1500 g) (29.1\%) and preterm $<32$ weeks (27.7\%). Significant maternal factors for NT included higher maternal age (33.3 years vs 31.6, $\mathrm{p}=0.02)$ and pre-eclampsia $(9.1 \%$ vs $3.9 \%, \mathrm{p}=0.02)$. Significant neonatal factors included being prematurity $<32$ weeks $(58.9 \%$ vs $2.6 \%, \mathrm{p}=0.000)$, birthweight $<1500 \mathrm{~g}(57.5 \%$ vs 19.9\%, $\mathrm{p}=0.000)$ and lower mean Apgar scores at birth $(p=0.000)$. Total of $41 / 73(56.1 \%)$ infants required platelet transfusions. NT that were transfused versus not transfused, other than maternal pre-eclampsia $(24.4 \%$ vs $12.5 \%$, $\mathrm{p}=0.242$ ), the rest of the factors listed above were found to be statistically significant.

For the first onset of NT, majority of transfused infants had early onset thrombocytopenia $(53.6 \%$ versus $62.5 \%$ in the non-transfused population, $\mathrm{p}=0.448)$. The mean day of onset of NT was 3.9 days in the transfused population and 2.9 days in the non-transfused population.

Majority had mild thrombocytopenia, not transfused, resolved in the first week of life. the predominant causes were mostly maternal factors pre-eclampsia, maternal Idiopathic Thrombocytopenia or IUGR secondary to placental factors. In infants whom were transfused, predominant factors included severe sepsis, especially gram-negative sepsis, Pulmonary Haemorrhage and NEC, Cytomegalovirus (CMV) infection, clinical sepsis and unknown cause. There were higher morbidities like IVH, BPD and ROP, death in transfused NT, but was not statistically significant difference. Only one infant required IVIG due to neonatal alloimmune thrombocytopenia. Conclusions The incidence of NT was $0.014 \%$, is higher at $29 \%$ in VLBW cohort and $28 \%$ of preterm born with GA of $<32$ Weeks. The significant maternal risk were pre-eclampsia and maternal thrombocytopenia. The significant Neonatal risk factors were asphyxia, lower GA, birth weight, SGA. In infants with thrombocytopenia that were severe and transfused, additional causes included severe sepsis, especially gramnegative sepsis, NEC and CMV infection

\section{TWELVE YEAR REVIEW OF GRADE 4 IVH (EXTENSIVE AND LOCALISED) WITH OUTCOMES}

Ashanti Sham Bala Krishnan, Robin Miralles. UK

\subsection{6/bmjpo-2021-RCPCH.135}

Background Intraventricular Haemorrhage (IVH) is bleeding into the ventricles and is common in preterm neonates. An increasing grade of IVH is associated with poorer neurological outcomes. Bilateral Grade $4 \mathrm{IVH}$ is associated with the poor prognosis and high rate of cerebral palsy. However recent research has shown that infants with unilateral grade 4 lesions had improved cognitive scores and better overall neurodevelopment outcomes.
Objectives To retrospectively assess outcomes at 2 years according to degree of severity of Grade $4 \mathrm{IVH}$ in preterm infants.

Methods Design: Single-centre tertiary neonatal unit. 64 infants admitted between 2006 and 2019 confirmed to have IVH with parenchymal infarction (Grade 4 IVH).

Method: Extent of infarction (PVHI) was further graded into 'localised' or 'extensive' (Volpe 2017). Two-year follow up for the inborn infants - Health Status Questionnaire, Schedule of Growing Skills, CP classified by site and GMFCS. Results Overall mortality 40/64 (63\%) - localised group 10/21 (48\%); extensive 30/43 (70\%).

Mortality was significantly higher in the extensive infarction group $(\mathrm{p}<0.05)$.

No infants with bilateral parenchymal infarction survived to discharge $(n=8)$.

Shunt placement in 4 (all extensive). 17/47 inborn survivors (8 extensive, 9 localised).

Two-year outcome data cognitive assessment was available for 10/17. Further information available on 4 others (not yet 24 months).

All infants with extensive infarction had CP (5/5). Localised infarction CP in $3 / 9 \quad(33 \%) \quad(p<0.05)$. Cognitive outcomes $(n=10)$ : Normal in $1 / 3$ of extensive group, 6/7 localised group.

Overall disability $(n=14)$ : Free of disability in localised group 5/9 (55\%), extensive: 0/5 (0\%).

Severe disability in localised group $0 / 9(0 \%)$, extensive $2 / 5$ $(40 \%)$

Conclusions Although having a Grade 4 IVH still carries a high mortality rate, significantly better outcomes were seen with a localised Grade $4 \mathrm{IVH}$, important information in making critical care decisions.

\section{IMPLEMENTING A TELEPHONE TRIAGE SERVICE FOR ACUTE GENERAL PAEDIATRIC REFERRALS FROM PRIMARY CARE IN A TERTIARY PAEDIATRIC HOSPITAL}

Clare Brady, Claire Gilmore, Lesley Nairm, Allison McKie, Owen Forbes. UK

\subsection{6/bmjpo-2021-RCPCH.136}

Background The General Paediatric Service at our Hospital is extremely busy, often with more than 70 inpatients under the team each day during months of peak activity and over 40 new acute telephone referrals from Primary Care daily. The number of children attending acute services at the hospital has been increasing annually and the Emergency Department will often have more than 300 attendances in 24 hours in winter months. This activity level means there is a significant pressure on inpatient beds and effective patient flow through the hospital is essential. During the ongoing COVID19 pandemic, there has been pressure on acute NHS services, which has driven the need for new innovative ways of working.

Objectives We aimed to ensure that all acute referrals to the General Paediatric Service were triaged via telephone by a senior paediatric clinician (registrar or consultant). The primary aim of this service was to ensure that children were streamed to the appropriate location, to be seen by the appropriate team at the appropriate time. Secondary aims included improving the interface with primary care and instigating prehospital management for patients streamed into outpatient services. 